\section{Les CDAG et la prise en charge de l'infection par le VIH}

Elisabeth Bouvet, Stéphane Le Vu dépistage anonyme et gratuit (CDAG) du VIH ont été mises en place dans chaque département, pour permettre une démarche individuelle et volontaire de dépistage de l'infection par le VIH. II existe actuellement plus de 380 CDAG dont les missions ont été régulièrement élargies par les autorités sanitaires. Elles doivent favoriser le dépistage précoce, faciliter l'accès au dépistage des personnes précarisées et des personnes vulnérables aux risques, renforcer la prévention en aidant les consultants à définir une stratégie personnelle de prévention vis-à-vis du VIH, mais aussi du VHC, de la syphilis et des autres maladies sexuellement transmissibles. Elles doivent aussi renforcer le lien entre dépistage et prise en charge. Les CDAG drainent une population jeune, donc plus à risque pour l'infection par le VIH que la population générale. Leur activité augmente régulièrement, et leur taux de positivité du dépistage est double de celui des laboratoires d'analyse médicale. Entre 1000 et 2000 diagnostics positifs d'infection par le VIH sont faits dans les CDAG chaque année, ce qui représentait $11 \%$ des sérologies positives à l'échelle nationale en 2002. La connaissance de l'impact réel des CDAG sur la prévention de l'infection par le VIH et leur rôle dans le dépistage sont limités par l'anonymat et le type de recueil d'informations volontairement réduit. Pour mieux connaître la typologie des consultants, le recueil des données d'activité va évoluer à partir de 2004 et sera complété par une enquête épidémiologique transversale et par la mise en place d'un réseau de CDAG assurant un recueil de données épidémiologiques plus complet et continu. <
En France, la politique de dépistage de l'infection par le virus de l'immunodéficience humaine (VIH) repose, depuis le début de l'épidémie, sur une démarche indivi-

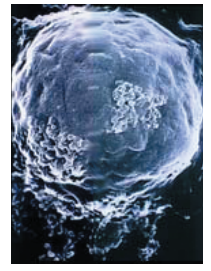

duelle et volontaire. Les consultations de dépistage anonyme et gratuit (CDAG) sont mises en place depuis 1988, au niveau de chaque département, dans des établissements de santé ou des dispensaires antivénériens. L'objectif initial était de faciliter le dépistage du VIH en supprimant les barrières liées au coût ou au risque de non confidentialité des résultats. Les CDAG principaux pouvaient disposer d'antennes, en particulier dans les prisons. Le dispositif gratuit a été étendu depuis 1992 aux dispensaires antivénériens (DAV), aux centres de planification et d'éducation familiales, ainsi qu'aux centres de protection maternelle et infantile (PMI). Dans ces structures, le dépistage est gratuit, mais non anonyme.

Les CDAG constituent la partie la plus visible de l'engagement de l'État dans la lutte contre la transmission du VIH. Elles représentent un outil original directement contrôlé et observé par l'État et se situent à côté des structures de prise en charge et de traitement de l'infection par le VIH, avec lesquelles elles ont des liens plus ou moins étroits.

Si l'activité des CDAG en termes de dépistage est mesurable, l'impact préventif de ces structures sur la transmission du VIH dans la population est difficile à appréhender. Cependant, des enquêtes sont régulièrement réalisées pour connaître les cibles, les motivations et l'aspect quantitatif des consultations. La nature anonyme du dispositif, composante fondamentale de la démarche, empêche de connaître certaines caractéris- 
tiques des consultants. C'est pourquoi des enquêtes transversales sont réalisées pour combler ce déficit d'information.

\section{Missions et objectifs des CDAG/DAV}

Les CDAG ont été initialement créés par le ministère chargé de la Santé pour permettre l'accès au dépistage, contribuer à la politique de prévention et favoriser la prise en charge des patients. Les CDAG sont désignés par le préfet. Les dépenses sont supportées par l'Assurance maladie pour $85 \%$, et par l'État pour $15 \%$. En 1998, une circulaire ministérielle (circulaire DGS/DH/DSS $\mathrm{n}^{\circ}$ 98-423 du 9 juillet ) [1] est venue définir de nouveaux objectifs pour les CDAG en raison de la transformation des stratégies thérapeutiques, des modifications des possibilités diagnostiques et de l'amélioration spectaculaire du pronostic vital des patients infectés traités par les trithérapies antirétrovirales. La compétence des consultations de dépistage du VIH a été étendue au dépistage du virus de l'hépatiteC (VHC), reconnu comme problème de santé publique majeur, puis au dépistage et à la prévention du virus de l'hépatite B (VHB), dont la transmission se fait principalement par voie sexuelle.

Cinq objectifs prioritaires ont alors été définis : (1) permettre une prise en charge précoce après exposition aux risques: il s'agit d'intervenir soit dans une indication de traitement après exposition, soit dans une phase de diagnostic de primo-infection. Cette action a pour objectif d'agir sur la phase initiale majeure de diffusion épidémique du VIH, de traiter le plus précocement possible et d'effectuer un travail de prévention centré sur la perception du risque. Les autres risques liés au VHB après exposition par voie sexuelle, et au VHC après contamination par voie sanguine, doivent également être pris en compte ; (2) rendre le dispositif visible pour tous en diffusant des informations destinées au public ; (3) faciliter l'accès au dépistage pour les personnes précarisées et les personnes vulnérables aux risques ; (4) renforcer la prévention en aidant les consultants à définir une stratégie personnelle de prévention vis-à-vis du sida, mais aussi de l'hépatite $C$, de la syphilis, et des autres maladies sexuellement transmissibles (MST) ; (5) renforcer le lien entre dépistage et prise en charge. Il est prévu de créer un accompagnement pour répondre aux divers problèmes, notamment la restauration des droits sociaux, l'information sur les traitements, la recherche d'un soutien dans l'entourage du patient et la facilitation de la prise de contact avec le réseau de soins.

Plus récemment, l'augmentation du nombre de diagnostics de syphilis en France, en particulier à Paris, a conduit les autorités sanitaires à renforcer le dépistage de cette maladie dans certaines populations à risque (homosexuels masculins et hétérosexuels multipartenaires) et à proposer la réalisation d'un dépistage de la syphilis dans les CDAG à l'occasion d'un passage ou d'une demande de test VIH. Ainsi, la demande de dépistage du VIH est utilisée comme moyen de sensibilisation aux risques de MST en général.
Ces missions doivent être effectuées par une équipe pluridisciplinaire comportant des médecins, des infirmières, des assistantes sociales, des psychologues, tous formés à l'accueil des consultants, l'éducation pour la santé, l'usage de substances psychoactives, la sexologie et l'infection par le VIH.

Ces actions doivent conduire à de bonnes pratiques de dépistage, s'articuler avec les partenaires locaux, en particulier les médecins généralistes et les biologistes, ainsi que les partenaires institutionnels (centres d'information et de soins de l'immunodéficience humaine [CISIH] et pôles de référence VHC). Une évaluation de l'activité de dépistage est effectuée par l'Institut national de veille sanitaire (InVS) sur la base de l'analyse des bilans trimestriels transmis par les centres.

\section{Population et recueil de données de l'activité des CDAG}

Chaque CDAG adresse un bilan d'activité trimestriel au médecin inspecteur de la DDASS (direction départementale des affaires sanitaires et sociales) qui le transmet à I'InVS après validation. L'analyse se fait sur des données agrégées sur l'année entière. La proportion de sujets testés positifs pour le VIH ne peut être calculée en raison de l'absence d'identification, un individu donné pouvant avoir subi plusieurs tests. Le résultat est donc exprimé en nombre de tests positifs pour 1000 tests effectués. Les dernières données publiées concernent les années 2001 et 2002 [2]. On recensait 363 CDAG en 2001 , et 386 en 2002 (hors des prisons) dont environ $80 \%$ avaient envoyé au moins un bilan trimestriel à l'InVS.

Le nombre d'hommes testés est supérieur au nombre de femmes (sex ratio 1,2), sauf chez les moins de 20 ans, où deux femmes pour un homme ont été testés. La tranche d'âge des 20-29 ans est la plus représentée: $54 \%$ des personnes appartenaient à cette classe. Globalement, $70 \%$ des personnes qui se présentent dans un CDAG ont moins de 30 ans. Dans une enquête transversale réalisée en 1999 [3], 94,7 \% des personnes ayant précisé leur nationalité étaient françaises. Parmi les $5,3 \%$ d'étrangers, $21 \%$ venaient d'Afrique du Nord et $29 \%$ d'Afrique sub-saharienne. Les personnes de nationalité étrangère étaient plus âgées, bénéficiaient beaucoup moins souvent de la sécurité sociale et semblaient être moins bien informées du risque de transmission du VIH.

La population ayant recours aux CDAG est différente de la population générale. Parmi les hétérosexuels, les consultants sont plus jeunes, souvent multipartenaires, utilisent moins les préservatifs, en particulier avec les partenaires occasionnels. Les homosexuels et les bisexuels sont plus représentés (11,2\% des hommes et $6,7 \%$ des consultants) que dans la population générale $(0,8 \%$ des hommes dans la population générale), et $4,2 \%$ des consultants se sont définis comme bisexuels.

La gratuité semble un élément très important de l'attractivité des CDAG pour la population étrangère. Dans cette population, la proportion de sujets n'ayant jamais été dépistés auparavant, 
qui découvraient leur infection au moment de l'entrée dans le stade sida, était plus importante chez les étrangers (56\%) que chez les français (38\%).

Enfin, les usagers de substances psychoactives sont peu nombreux à fréquenter les CDAG.

Ainsi, les CDAG sont identifiés comme étant des lieux de recours au test dans un contexte de risque sexuel. Plus de la moitié des personnes interrogées avaient déjà réalisé un test. Dans la même enquête transversale de 1999 [3], chez les personnes déjà testées, une moyenne de 2,26 tests avait été réalisée. Près de la moitié des consultants désiraient un test en raison d'une inquiétude à la suite d'un rapport sexuel, $27 \%$ avaient répondu au souhait de leur partenaire, $21 \%$ avaient voulu faire le test avant d'arrêter d'utiliser des préservatifs, et 11,6\% après un incident de préservatif. La plupart des hommes homosexuels étaient venus au CDAG après un rapport qui les avait inquiétés. Les femmes demandaient plus souvent aux hommes de faire un test. L'analyse des correspondances multiples a permis de distinguer trois classes parmi les personnes n'ayant jamais subi de test : un premier groupe ( $60 \%$ de l'effectif) dans lequel les sujets n'avaient pas peur de la contamination et venaient souvent en couple avant d'arrêter le préservatif ; un deuxième groupe (17\%) comprenant des personnes ayant peur d'une contamination et doutant de la fidélité de leur partenaire. Le test était parfois réalisé car le partenaire refusait de faire un test ; un troisième groupe (23\%) comportait des individus craignant une contamination sans mentionner de risque précis : ces sujets utilisaient peu le préservatif, avaient de nombreux partenaires occasionnels et venaient le plus souvent consulter seuls.

On retrouvait les trois mêmes classes chez les personnes déjà testées auparavant, mais les proportions étaient différentes : près de $50 \%$ pour la première classe, $33 \%$ pour la seconde et $21 \%$ pour la troisième.

Cette enquête épidémiologique transversale a été répétée en 2000 et n'a pas montré de modification dans la répartition du recrutement.

\section{Bilan de l'activité de dépistage}

En 2002, on recensait 495 CDAG dont 109 dans les prisons. En 2002, le nombre de tests VIH dans les CDAG a été de 251857 , chiffre stable par

\begin{tabular}{llcccc}
\hline $\begin{array}{l}\text { Groupe } \\
\text { de transmission }\end{array}$ & $\begin{array}{l}\text { Origine } \\
\text { géographique }\end{array}$ & n & $\%$ & $\mathbf{n}$ & $\%$ \\
\hline Homosexuels & France & 99 & 33 & 98 & 26 \\
& Pays étranger & 55 & 18 & 62 & 16 \\
\hline Hétérosexuels & France & 23 & 7 & 14 & 4 \\
& Pays étranger & 101 & 33 & 172 & 46 \\
Toxicomanes & Toutes & 9 & 3 & 7 & 2 \\
Inconnus & France & 8 & 3 & 3 & 1 \\
& Pays étranger & 8 & 3 & 17 & 5 \\
Total & & 303 & 100 & 373 & 100 \\
\hline
\end{tabular}

Tableau I. Caractéristiques épidémiologiques des sujets positifs pour le VIH, testés au CDAG de Paris, évolution 1998-2001 (d'après [5]). rapport aux années précédentes [2]. Par ailleurs, une étude conduite par l'InVS a permis d'estimer que 4,5 millions de sérologies VIH avaient été réalisées en France dans les laboratoires d'analyse médicales la même année [4]. La part du dispositif gratuit (CDAG/DAV) représentait donc $6 \%$ de l'ensemble des tests, hors dons du sang. La proportion de diagnostics positifs était de 5 pour 1000 en 2002 dans les CDAG [2] et de 2,6 pour 1000 hors CDAG [4], ce qui montre que les consultants de CDAG sont plus à risque que la population testée dans les laboratoires.

Une tendance globale à l'augmentation des diagnostics positifs a été observée entre 1999 et 2001 , mais ne s'est pas confirmée en 2002. Par ailleurs, et pour la première fois en 2002, le taux de positivité chez les femmes de 30 à 39 ans a dépassé celui des hommes de la même tranche d'âge. Une augmentation des diagnostics positifs a été observée à Paris avec un taux de 12,8 pour 1000 en 2002 contre 11,2 pour 1000 en 2001 . Le nombre et la proportion de diagnostics positifs sont restés stables chez les homosexuels, à Paris, entre 1998 et 2001 (Tableau I) [5]. En revanche, le nombre de diagnostics positifs chez les personnes contaminées lors de pratiques hétérosexuelles a augmenté. Cette augmentation est observée essentiellement chez les femmes originaires d'un pays étranger, particulièrement d'Afrique sub-saharienne. Malheureusement, le système de surveillance ne permet pas de distinguer les infections récentes des infections anciennes. II n'est donc pas possible de savoir si cette augmentation reflète un afflux de personnes étrangères dans les CDAG à des fins de dépistage. Cependant, cette information est à rapprocher de l'augmentation de l'incidence du sida dans cette population en France. Les CDAG jouent donc probablement un rôle significatif dans l'accès au système de santé français pour ces populations marginalisées. Les CDAG permettent un test gratuit et la possibilité de consulter un médecin spécialisé. Cependant, les suites d'un diagnostic positif sont mal connues. Combien de personnes dépistées positives consultent effectivement pour leur infection par le VIH, combien sont prises en charge, et comment cette information est-elle acceptée et répercutée dans le milieu familial ou communautaire? Toutes ces questions font l'objet d'enquêtes complémentaires dans les populations concernées. De plus, la déclaration obligatoire des nouveaux diagnostics d'infection par le VIH devrait également apporter des éléments de réponse à cette question, malgré la non-participation des CDAG au système de surveillance. II convient certainement d'adapter les structures à cette modification du recrutement. 


\section{Conclusions}

Les CDAG effectuent $6 \%$ des tests de dépistage du VIH en France, et rendent, chaque année, 1000 à 2000 diagnostics positifs. La proportion de tests positifs est plus élevée que dans les autres structures de dépistage. Les CDAG drainent en effet une population jeune et plus à risque que la population générale pour l'infection par le VIH. On peut donc en conclure que l'objectif d'incitation au dépistage des populations les plus à risque est atteint, du moins en partie. Cependant, la nature volontairement limitée des informations recueillies ne permet ni de connaître l'impact réel des CDAG sur la prévention de la transmission du VIH, ni les filières suivies par les sujets après leur dépistage. Des structures de même type ont été mises en place dans d'autres pays, en particulier dans les pays à forte prévalence, sous l'impulsion de l'Organisation mondiale de la santé. Toutefois, on ne dispose pas d'études permettant de mesurer l'impact réel de ces structures sur la précocité du dépistage par rapport aux autres sites pratiquant ce type de tests. Une étude réalisée aux États-Unis montre que le dépistage anonyme est associé à une prise en charge plus précoce [6]. On ne dispose pas d'études permettant de savoir quel est l'impact de l'anonymat sur le taux de dépistage en France. II n'est pas prouvé que le caractère anonyme du test soit un argument décisif pour faciliter la réalisation du test dans la population à risque.

Pour mieux déterminer les caractéristiques des consultants, le recueil des données d'activité va évoluer en 2004 et sera complété par une enquête épidémiologique transversale et la mise en place d'un réseau de CDAG assurant un recueil de données épidémiologiques permanent et plus complet [2].

II n'est pas exclu que l'impact des CDAG dans le dépistage et la prévention soit très différent selon les régions. Ainsi, la région Provence-Alpes-Côte d'Azur, qui était très concernée historiquement par l'infection VIH, présente un taux de positivité en CDAG plus faible qu'au niveau national, ce qui ne peut être expliqué par les seules données d'activité.

Pour conclure, il convient d'insister sur le fait que le concept de CDAG à la fois anonyme et gratuit est en plein questionnement. II est vraisemblable que les données apportées par les enquêtes à venir aideront à faire évoluer la définition de ces structures. Faut-il lier anonymat et gratuité? Comment permettre une adaptation aux populations les plus concernées que sont les personnes d'origine étrangère? Comment faciliter le lien avec les structures de prise en charge de l'infection par le VIH? Enfin, les CDAG doivent-ils évoluer dans leur organisation et leur localisation pour aider à un dépistage plus précoce des groupes à risque? $\diamond$

\section{SUMMARY}

Free anonymous screening consultation and clinical management of HIV infection

In France, since 1988, anonymous and free consultations (called CDAG) have been settled in the country to facilitate an individual and volunteer approach for HIV screening. At the time, there are more than 380 CDAG with newly defined objectives. CDAG are supposed to encourage early screening, facilitate access to precarious persons and persons at risk for sexually transmitted diseases, and reinforce prevention, helping consultants to define a personal preventive strategy. CDAG are also supposed to play a role in prevention of both hepatitis B and C, and syphilis. They may help to link screening and healthcare. Their activity is increasing and the rate of positive test is twice that of private laboratories. Patients consulting those facilities are younger and more at risk than general population. Between 1000 and 2000 HIV positive tests are detected in CDAG each year ( $11 \%$ of positive tests in the country). The real impact on prevention and screening at the national level is unknown, in part because of anonymity. To improve the characterization of consultants, data collection will be modified in 2004, and a network of selected and volunteer centres will collect continuously more accurate data. $\diamond$

\section{RÉFÉRENCES}

1. Circulaire DGS/DH/DSS n $98-423$ du 9 juillet 1998 relative aux missions et aux objectifs des consultations de dépistage anonyme et gratuit ou de dépistage gratuit du virus de l'immunodéficience humaine. Bulletin Officiel 1998, n98/30.

2. Le Vu S, Herida M, Pillonel J, et al. Consultations de dépistage anonyme et gratuit (CDAG). Bilan 2001 et 2002 d'activité du dépistage VIH en France. BEH 2004 ; 17 : 65-6.

3. Gouezel P. Dispositif de dépistage anonyme et gratuit du VIH. Un regard sur les consultants. Enquête nationale juin-juillet 1999. Saint-Maurice : Institut national de Veille Sanitaire, novembre 2000.

4. Cazein F, Lot F, Couturier S, Semaille C. Activité de dépistage du VIH en 2001 et 2002 dans les laboratoires d'analyse. VIH, sida et IST.

Saint-Maurice : Institut national de Veille Sanitaire, 27 novembre 2003 (http://www.invs.sante.fr/publications/2003/vih_sida_ist_2003/ depistage_vih_2002.pdf).

5. Gouezel P, Simon A, Derouineau J, et al. Consultations de dépistage anonyme et gratuit (CDAG), bilan d'activité du dépistage de VIH, Paris, évolution 1998-2001. BEH 2002 ; 43 : 2156

6. Bindman AB, Osmond D, Hecht FM, et al. Multistate evaluation of anonymous HIV testing and access to medical care. Multistate evaluation of surveillance of HIV (MESH) study group. JAMA 1998 ;

$280: 1416-20$.

\section{TIRÉS À PART}

$\varepsilon$. Bouvet 